\title{
Yb-fiber amplifier pumped idler-resonant PPLN optical parametric oscillator producing 90 femtosecond pulses with high beam quality
}

\author{
Lin Xu*, James S. Feehan, Li Shen, Anna C. Peacock, David P. Shepherd, David J. Richardson \\ and Jonathan H.V. Price \\ Optoelectronics Research Centre, University of Southampton, Southampton SO17 1BJ, UK \\ *Corresponding author: l.xu@soton.ac.uk fax: 02380593142
}

\begin{abstract}
An idler-resonant femtosecond optical parametric oscillator (OPO) with near-diffraction-limited beam quality $\left(M^{2} \sim 1.05\right)$ at $\sim 2.4 \mu \mathrm{m}$ is demonstrated. The OPO is synchronously pumped by a femtosecond Yb:fiber amplifier system providing 130 fs pulses with an average power of $4.5 \mathrm{~W}$ at $1050 \mathrm{~nm}$ and delivers 90 fs pulses with maximum average powers of $600 \mathrm{~mW}$ for the idler and $670 \mathrm{~mW}$ for the signal. Tunability, is demonstrated across idler (signal) wavelengths from 2.2-2.6 $\mu \mathrm{m}(1.76-2.0 \mu \mathrm{m})$, limited only by the OPO mirrors. As a demonstration of the utility of the source, the idler pulses are used to generate a supercontinuum from $1600 \mathrm{~nm}$ to $3200 \mathrm{~nm}$ in a silicon-core fiber.
\end{abstract}

\section{Introduction}

Femtosecond (fs) laser sources in the mid-infrared (mid-IR) range are important for spectroscopy as well as a range of other applications [1,2]. Due to the lack of commercially available mid-IR resonant gain media, there have been only a few reports of fs-lasers developed beyond a wavelength of $2.5 \mu \mathrm{m}$ [3,4]. Although $2 \mu \mathrm{m}$ fiber lasers have been developed in the fs regime [5], to go further into the mid-IR requires frequency conversion. Different frequency generation (DFG) is an option [6], but typically has low efficiency Synchronously pumped optical parametric oscillators (OPOs) are recognized as useful laser sources for the production of tunable output pulses over a broad wavelength range including the mid-IR [7-9]. This includes phase-stabilised OPOs extending the frequency comb of carrier-envelope-offset locked pump lasers to enable high precision spectroscopic studies [10-12]. The most commonly used OPO gain material in such systems is MgO-doped periodically-poled lithium niobate (PPLN). Orientation patterned GaAs (OP:GaAs) has also been used [13,14] but it is not yet as widely available as PPLN. It should be noted that all of the references above were signal resonant cavities.

In parallel with OPO based spectroscopy, there has been interest in the potential for waveguides in both planar and fiber geometries to provide extremely broadband mid-IR spectral output via nonlinear broadening or supercontinuum generation seeded by a fixed wavelength femtosecond pulse source. Supercontinuum spectra in the mid-IR are of interest due to their large bandwidth and high brightness, which could, for example, enable high-throughput chemical screening [2]. Simulation results and experimental work show that non-silica fibers can produce continuum spectra in the range from 3.0 to $5.0 \mu \mathrm{m}$ [15-18], with potential for extension to longer wavelengths using certain glasses (e.g. chalcogenides). The generation of high coherence mid-IR continuum, dominated by self-phase modulation, is of particular interest and simulations indicate that this should be achievable from seed sources in the 2.2-2.5 micron region [17]. This is in contrast to the more widely used schemes for generating low-coherence supercontinuum, which typically rely on a combination of self-phase modulation, four-wave mixing, the Raman response function, soliton fission etc. There is also growing interest in nonlinear mid-IR photonics in silicon waveguides, where at wavelengths $>2.2 \mu \mathrm{m}$ the two-photon absorption coefficient drops to zero [1921]. Advancing these research areas ideally requires femtosecond pulse sources in the 2.2-2.5 micron region with diffractionlimited beam quality (for coupling into single-mode waveguides and fibers). However, in signal-resonant OPOs, the idler is not constrained by the cavity mirrors and non-diffraction-limited outputs may be obtained [22]. In this paper we report the development of a femtosecond idler-resonant OPO system pumped by an Yb-fiber laser that is targeted towards such studies.

While the fundamental physics of nonlinear gain and material transparency underpin the performance of both signal- and idler-resonant PPLN OPOs, nonetheless, there are significant differences in the operation of idler resonant OPOs because the 
resonating idler wave is subject to the effects of the PPLN refractive index, gain-bandwidth and dispersion at longer wavelengths and commercially available PPLN crystals currently do not have optimised anti-reflection coatings for the idler. Furthermore, the atmospheric absorption can be a more important factor for idler resonant OPOs than for signal resonant OPOs and was observed to prevent operation in our system in the relevant wavelength bands unless the OPO enclosure was nitrogen-purged. We are not aware of reports of fs, singly resonant PPLN OPOs with circulating idler and have therefore characterized this source in detail. In signal-resonant PPLN-based cavities in particular, it is possible to use a single mirror set to cover the entire tuning range. This is limited on the short wavelength side for the signal and, correspondingly, on the long wavelength side for the idler, by the onset of absorption at wavelengths above $\sim 4.5 \mu \mathrm{m}$. However, we found that optical coating materials cannot provide a single mirror set covering the entire idler tuning range of the PPLN crystal and we have selected mirrors covering the 2.2-2.6 micron region for the work reported here. As described below, the resulting OPO generated $600 \mathrm{~mW}$ of idler power with high beam quality, wavelength tunable from 2.2-2.6 $\mu \mathrm{m}$ and pulse durations below $100 \mathrm{fs}$. The OPO cavity length was also actively stabilized against thermally induced fluctuations to enable robust operation. As a demonstration of the utility of the sources, the idler pulses were used to pump a silicon-core fiber and generate a 1600 $\mathrm{nm}$ to $3200 \mathrm{~nm}$ supercontinuum.

\section{Experimental setup and result}

\subsection{OPO system}

The experimental setup of the femtosecond OPO is shown in Fig. 1. The master-oscillator-power-amplifier (MOPA) pump source consisted of a Nd:glass mode-locked seed laser (GLX-200, Time-Bandwidth Ltd.), operating at a repetition rate of $80 \mathrm{MHz}$, with pulse duration of $200 \mathrm{fs}$, average power of $120 \mathrm{~mW}$ and central wavelength of $1050 \mathrm{~nm}$, followed by an $\mathrm{Yb}$ fiber amplifier. The amplifier consisted of a 3-m-long polarization maintaining large-mode-area Yb fiber (PLMAYDF-25/250-VIII from Nufern), which had a core diameter of $25 \mu \mathrm{m}$ with a numerical aperture (NA) of 0.06 and cladding diameter of $250 \mu \mathrm{m}$ with a NA of 0.46 , pumped by a fiber pig-tailed 975-nm laser diode. The input end of the fiber was tapered and spliced with a short piece of passive single-mode fiber (PM980 - Nufern) to maintain fundamental-mode operation. The amplifier was operated in the parabolic pulse regime [23]. A transmission-grating-based compressor finally provided $130 \mathrm{fs}$ pulses with average power of $4.5 \mathrm{~W}$, linear polarization and $M^{2} \sim 1.08$. The spectrum is similar to that of the undepleted pump shown in Fig. 2.(a). The power was varied using the half-wave plate before the polarizing isolator shown in Fig. 1. in order to avoid changing the pump spectrum and pulse duration, as would otherwise have occurred if the pump power of the amplifier was changed.

The OPO cavity comprised four plane mirrors and two concave spherical mirrors (300-mm radius of curvature). All six mirrors were coated for high transmission (HT) over the range from 1020-1080 nm (T > 90\%) and 1630-2050 nm (T > $78 \%$ ), and high reflectivity (HR) across the range from $2200-2700 \mathrm{~nm}(\mathrm{R}>99 \%)$. The fundamental repetition rate of the OPO cavity was matched to the pump source, leading to a total cavity length of $3.64 \mathrm{~m}$. To allow fine tuning of the cavity length the plane mirror, M6, was set on a piezo translation stage, providing $5 \mathrm{~nm}$ resolution across the $20 \mu \mathrm{m}$ of available travel.

The output from the Yb-fiber MOPA source was focused into a periodically poled 5\% MgO-doped $\mathrm{LiNbO}_{3}$ (PPLN) crystal with a $1 / \mathrm{e}^{2}$ intensity beam waist radius of $75 \mu \mathrm{m}$ and a peak intensity of $4.6 \mathrm{GW} / \mathrm{cm}^{2}$, which is close to the quoted damage threshold. The calculated beam waist of the resonated idler was matched to the pump focal spot size by separating the two curved mirrors by a distance of $315 \mathrm{~mm}$. The 1-mm-long PPLN crystal (MOPO1-1.0-1 from Covesion Ltd.), had five gratings with periods ranging from 29.5 to $31.5 \mu \mathrm{m}$ in steps of $0.5 \mu \mathrm{m}$ and was mounted inside an oven to allow temperature tuning in the range from $20^{\circ} \mathrm{C}$ to $200{ }^{\circ} \mathrm{C}$ with a precision of $0.1{ }^{\circ} \mathrm{C}$. The signal and residual pump were measured after CM2. The idler output was extracted using a pellicle beam splitter inserted between M5 and M6. The reflectivity of the pellicle could be angle-tuned from approximately $28 \% \sim 46 \%$ at the idler wavelength. Our measurements showed that the maximum output power was obtained for an idler wavelength of $2.44 \mu \mathrm{m}$ using the $31.5 \mu \mathrm{m}$ grating, a crystal temperature of $100^{\circ} \mathrm{C}$ and with the pellicle tuned to provide $\mathrm{R}=30 \%$. This setup led to a threshold power for the OPO of $2.0 \mathrm{~W}$, so the 
OPO could be operated up to 2.2 times threshold. The residual pump spectra were measured with the OPO cavity blocked (undepleted pump) and oscillating (depleted pump) and are also shown in Fig. 2.(a). The calculated pump depletion is the difference between undepleted and depleted pump spectra.

\subsection{OPO Characterisation}

Fig. 2.(b) shows the signal and idler output powers versus the pump power at an idler wavelength of $2.4 \mu \mathrm{m}$. The output powers increased linearly with pump power and the slope efficiencies for the signal and idler beams, $\eta_{\mathrm{s}}$ and $\eta_{\mathrm{i}}$, were $26 \%$ and $23 \%$, respectively, reaching maximum powers of $670 \mathrm{~mW}$ and $600 \mathrm{~mW}$ at the full $4.5 \mathrm{~W}$ of pump power. As this is close to the quoted damage threshold, this represented the limit on power scaling of our system without changing the spot sizes within the PPLN crystal. The overall power conversion efficiency was $28 \%$. The off-the-shelf PPLN crystal was supplied with a standard anti-reflection (AR) which was optimized for typical signal wavelengths ( $R<1 \%$ at $1050 \mathrm{~nm}$ and $1500-1700 \mathrm{~nm}$ ) but had a reflectivity of approximately $\sim 3.5 \%$ on each surface at the idler wavelengths generated here. Therefore the intra-cavity loss of the resonated idler is significant when compared to the output coupling fraction. We should note that custom AR coatings are available that could be optimized for our idler wavelength range, thus these losses are not a prohibitor for future high power systems. To avoid the formation of a sub-cavity, the crystal was tilted by a small angle from the pump input direction.

For a fixed grating with a period of $31.5 \mu \mathrm{m}$ and a temperature of $100{ }^{\circ} \mathrm{C}$, the output power and wavelength of the signal and idler could be tuned by changing the OPO cavity length, as shown in Fig. 3 . The idler shifted to a shorter wavelength on decreasing the cavity length in order to maintain synchronous operation of the OPO, because the delay of idler pulses with respect to pump pulses became smaller as explained in [24]. The wavelength of the idler decreased when decreasing the cavity length. The zero relative cavity length is chosen as the position where the threshold of the OPO was the minimum. The total M6 mirror movement possible before the OPO ceased to oscillate was $\sim 13 \mu \mathrm{m}$ (i.e. cavity detuning length of $\sim 26 \mu \mathrm{m}$ ). The average tuning sensitivity across the operating range of the $\mathrm{OPO}$ was about $6 \mathrm{~nm} / \mu \mathrm{m}$. The asymmetry in the output power versus cavity length detuning is similar to that in other experimental observations [24]. Cheng-Liu et al. have proposed that this is due to intensity-dependent and detuning dependent phase-shift terms in the expression for the gain [25]. It was not initially possible to tune the idler wavelength above $2.5 \mu \mathrm{m}$, due to strong water vapor absorption in the cavity [9]. In order to achieve idler pulses at longer wavelengths, the OPO cavity was enclosed and purged with dry nitrogen. In this case, the $31.0 \mu \mathrm{m}$ period grating was chosen and the temperature was moved close to $140{ }^{\circ} \mathrm{C}$. The spectrum of the shortest signal wavelength achieved was $1.76 \mu \mathrm{m}$, indicating the longest achievable idler wavelength is about $2.6 \mu \mathrm{m}$.

When investigating the idler pulse duration with an autocorrelator (pulseCheck, APE Ltd.) we observed the shortest pulses at around $2.4 \mu \mathrm{m}$. Fig. 4. shows how the idler-pulse duration changed with wavelength. The inset to Fig. 4. shows the calculated total intra-cavity group delay dispersion (GDD) and the GDD from the six cavity mirrors and the PPLN crystal at $100{ }^{\circ} \mathrm{C}$ [26]. There is a discrepancy in the observed behavior compared to that expected. Considering the wavelength tuning shown in Fig. 3., the monotonic shift in wavelength with cavity length implies that the GDD does not cross zero (i.e. the group delay is monotonic) whereas according the our prediction in Fig. 4., the GDD has a zero-dispersion-wavelength (ZDW) at $\sim 2.5 \mu \mathrm{m}$. A ZDW in the operating range has been observed by others to lead to two wavelengths oscillating simultaneously in the cavity with equal offset from the ZDW [27]. We did not observe such behavior. It is possible that small departures from the dispersion parameters predicted from the Sellmeier data [26] could explain these observations as our femtosecond system will be highly sensitive to dispersive effects. Such discrepancies in the dispersion could arise due to variations in the material composition and also the particular choice of equation chosen for the Sellmeier fit, which would result in a difference between the calculated and actual ZDW of our crystal. However, in this paper we have chosen to focus on the experimentally observed behaviour of the OPO, and therefore just note this difference following the approach of the report by [27].

With the idler wavelength centered at $2.4 \mu \mathrm{m}$, the autocorrelation of the shortest pulse was recorded and the spectrum was measured with a monochrometer (TMc300, Bentham Ltd.), as presented in Fig. 5. We believe the high frequency 
modulation observed across the idler spectrum is due to the multi-mode fiber used to collect the light. The deconvolved fullwidth-half-maximum (FWHM) pulse duration was 90 fs (assuming a Gaussian pulse shape). The idler pulse was therefore somewhat shorter than the pump pulse. The FWHM spectral bandwidth was approximately $100 \mathrm{~nm}$ (time-bandwidth product of 0.46 ), so the pulse duration is close to the transform limit. We note that when the pump is longer than the idler, the system operates somewhat like a gain-switched laser to shorten the idler pulse. Once the idler becomes shorter than the pump, as observed here, we expect the minimum pulse duration to be controlled by the intra-cavity dispersion, by the gainbandwidth of the PPLN crystal and by the walk-off of pump, signal and idler pulses. However, as noted above, it is not possible to use the PPLN Sellmeier data [26] to reliably analyze the OPO behavior in detail in this spectral region.

The beam quality of the OPO is of crucial importance in many intended end-applications of the source. In this work, the beam quality of both the signal and idler were characterized with a Pyrocam-based profiler (NanoScan, Photon Inc.). Fig. 6. shows the measured beam quality of the idler at around $2.4 \mu \mathrm{m}$ with $M_{x}^{2}=1.07$ and $M_{y}^{2}=1.03$ in the horizontal and vertical planes, respectively, at the maximum output power. The PPLN absorption is low across the OPO tuning range reported here and hence we would not expect thermally induced distortions to degrade the mode quality for this system [28]. The inset to Fig. 6. shows the idler beam profile in the far field. The comparable data for the signal was $M_{x}^{2}=1.33$ and $M_{y}^{2}=1.39$. As expected, the resonated wave in this singly resonant OPO maintained a better beam quality than the non-resonated wave, benefiting from the constraint provided by the cavity [22,29].

In our system, we were mainly interested in stabilizing the cavity against thermal drifts and hence used a PC based LabView program to control a piezo stage supporting one of the cavity mirrors (M6). The update speed of the system was therefore limited to $\sim 10 \mathrm{~Hz}$. This contrasts with the requirement in frequency-comb OPO systems, which have used $\mathrm{kHz}$ bandwidth feedback loops to lock the OPO cavity to the repetition frequency of the pump laser. We chose the sum-frequency wavelength of the idler and the pump, which was generated at low levels within the OPO, as the feedback parameter for this servo-loop [30]. After a dielectric mirror (M7) had filtered out the residual pump, the red sum-frequency light was reflected by a long-pass-filter and dispersed by a 600 lines/mm grating onto a position-sensitive detector (PDQ80A from Thorlabs Ltd.). This then provides a wavelength-dependent output voltage that can be used for feedback control of the cavity length. A slit was used before the position-sensitive detector to remove light generated by other nonlinear processes within the PPLN (e.g. sum frequency of the pump and signal wavelengths). The cavity stability was confirmed by recording the idler output power with the servo loop operating and disabled, respectively, over a 20 minute period with the results shown in Fig. 7. When the servo-loop was disabled, the idler output power gradually changed with time, as would be expected with thermal fluctuations and considering the variation of idler power with detuning of the cavity length. When the OPO servoloop is operating, the idler power exhibited excellent stability with less than $1 \%$ variation over 20 minutes. Although the feedback loop is not fast enough for compensating for mirror vibrations and unsuitable for a frequency comb system, we observed stable autocorrelation traces and stable oscilloscope output from the monitor photodiode which provided anecdotal evidence that the optical table and enclosure used to protect the OPO acted to reduce these effects on the output power to $\sim 1 \%$ fluctuations. We also monitored the wavelength of the idler on an optical spectrum analyser and observed similarly small fluctuations in the central wavelength.

\subsection{Supercontinuum generation}

Our exemplar supercontinuum experiment involved using the OPO idler to pump a short piece of hydrogenated amorphous silicon fiber, which has been shown to have the desirable combination of a large nonlinear refractive index and a negligible nonlinear (two photon) absorption parameter for wavelengths beyond $2 \mu \mathrm{m}$ [21]. The applications of nonlinear silicon photonics range from broadband telecommunications to sensing and spectroscopy, and the advantages of silicon-core fibers for such applications have been reported elsewhere [31]. The silicon fiber used in this experiment had a core diameter of 1.7 $\mu \mathrm{m}$, so that the zero dispersion wavelength is positioned around $2.1 \mu \mathrm{m}$. To ensure we were pumping well within the anomalous dispersion regime, where we expect the broadest supercontinua to be generated, the OPO was tuned out to 2.4 $\mu \mathrm{m}$. The incident beam was focused into the 4-mm-long silicon fiber using a 60x objective lens and the output collected via 
two ZnSe lenses to couple into a 1-m-long multimode indium fluoride fiber with high transmission over the 0.3-5.5 $\mu \mathrm{m}$ spectral range. The fiber was then connected to a grating-based monochromator (Bentham TMc300) to analyze the output spectra. The near-diffraction-limited quality of the idler beam allowed for preferential excitation of the fundamental mode of the multi-mode silicon fiber [31]. An incident average power of $15 \mathrm{~mW}$, measured after the focusing lens, resulted in a large spectral broadening as shown in Fig. 8. The full spectrum spanned from $1600 \mathrm{~nm}$ to $3200 \mathrm{~nm}$, which is the broadest supercontinuum generated in a silicon core fiber, to the best of our knowledge. The power was not increased beyond this point due to concerns over damaging the fiber. A full presentation of the fiber characteristics and an investigation of fourwave mixing and supercontinuum generation within the fiber will be reported elsewhere.

\section{Conclusions}

In conclusion, we have demonstrated a synchronously pumped, femtosecond, idler-resonant PPLN OPO, pumped by a femtosecond Yb:fiber MOPA, for applications such as mid-IR supercontinuum generation. With the enclosure purged using dry nitrogen, the system generated signal and idler pulses with wavelengths tunable in the range 1.76-2.0 $\mu \mathrm{m}$ and 2.2$2.6 \mu \mathrm{m}$, respectively, with tunability limited by the availability of broadband idler mirrors. The OPO produced a signal average power of $670 \mathrm{~mW}$ and an idler average power of $600 \mathrm{~mW}$. The idler-resonant cavity ensures that the idler has near diffraction-limited beam quality $\left(\mathrm{M}^{2} \sim 1.05\right)$ suitable for efficient launching into optical fibers. The shortest pulses, with a duration of $90 \mathrm{fs}$, were measured at a wavelength of $2.4 \mu \mathrm{m}$ and the pulses were close to transform limited. Active stabilization of the OPO cavity length against thermally induced fluctuations was achieved with a servo-loop controlling one of the cavity mirror positions and hence long-term power fluctuations were reduced to $\sim 1 \%$. Finally, we demonstrated the application of this source to mid-IR supercontinuum generation in non-silica fibers by pumping a silicon-core fiber and generating a supercontinuum from 1.6 to $3.2 \mu \mathrm{m}$.

\section{Acknowledgements}

This work has been partly funded by EPSRC UK grants EP/H049371/1 and EP/I02798X/1. We would like to acknowledge valuable discussions with Shaif-ul Alam (ORC, Southampton), Alexander Heidt (ORC, Southampton), Florian Emaury (currently at ETH, Zurich) and Hazel Hung (Covesion Ltd) and assistance from Ho Yin Chan (ORC, University of Southampton). 


\section{References}

1. S. Woutersen, U. Emmerichs, and H. J. Bakker: Science 278, 658 (1997).

2. C. F. Kaminski, R. S. Watt, A. D. Elder, J. H. Frank, and J. Hult: Appl. Phys. B 92, 367 (2008).

3. N. Tolstik, E. Sorokin, and I. T. Sorokina: Opt. Express 22, 5564 (2014).

4. M. N. Cizmeciyan, H. Cankaya, A. Kurt, and A. Sennaroglu: Opt. Lett. 34, 3056 (2009).

5. Y. Nomura and T. Fuji: Opt. Express 22(2014).

6. C. Erny, K. Moutzouris, J. Biegert, D. Kühlke, F. Adler, A. Leitenstorfer, and U. Keller: Opt. Lett. 32(2007).

7. K. A. Tillman, D. T. Reid, D. Artigas, and T. Y. Jiang: J. Opt. Soc. Am. B 21, 1551 (2004).

8. M. V. O'Connor, M. A. Watson, D. P. Shepherd, D. C. Hanna, J. H. V. Price, A. Malinowski, J. Nilsson, N. G. R. Broderick, D. J. Richardson, and L. Lefort: Opt. Lett. 27, 1052 (2002).

9. N. Coluccelli, H. Fonnum, M. Haakestad, A. Gambetta, D. Gatti, M. Marangoni, P. Laporta, and G. Galzerano: Opt. Express 20, 22042 (2012).

10. F. Adler, K. C. Cossel, M. J. Thorpe, I. Hartl, M. E. Fermann, and J. Ye: Opt. Lett. 34, 1330 (2009).

11. N. Coluccelli, A. Gambetta, T. Sala, D. Gatti, M. Marangoni, P. Laporta, and G. Galzerano: Opt. Lett. 37, 4440 (2012).

12. J. Mandon, Y. Jin, S. M. Cristescu, and F. J. M. Harren, in CLEO: 2013, OSA Technical Digest (online) (Optical Society of America, 2013), CTu3H.4.

13. M. W. Haakestad, T. P. Lamour, N. Leindecker, A. Marandi, and K. L. Vodopyanov: J. Opt. Soc. Am. B 30 , 631 (2013).

14. N. Leindecker, A. Marandi, R. L. Byer, K. L. Vodopyanov, J. Jiang, I. Hartl, M. Fermann, and P. G. Schunemann: Opt. Express 20, 7046 (2012).

15. P. Domachuk, N. A. Wolchover, M. Cronin-Golomb, A. Wang, A. K. George, C. M. B. Cordeiro, J. C. Knight, and F. G. Omenetto: Opt. Express 16, 7161 (2008).

16. J. H. V. Price, T. M. Monro, H. Ebendorff-Heidepriem, F. Poletti, P. Horak, V. Finazzi, J. Y. Y. Leong, P. Petropoulos, J. C. Flanagan, G. Brambilla, F. Xian, and D. J. Richardson: IEEE. J. Sel. Topics Quantum Electron. 13, 738 (2007).

17. J. H. V. Price, X. Feng, A. M. Heidt, G. Brambilla, P. Horak, F. Poletti, G. Ponzo, P. Petropoulos, M. Petrovich, J. Shi, M. Ibsen, W. H. Loh, H. N. Rutt, and D. J. Richardson: Optical Fiber Technology 18, 327 (2012).

18. J. Hu, C. R. Menyuk, L. B. Shaw, J. S. Sanghera, and I. D. Aggarwal: Opt. Lett. 35, 2907 (2010).

19. X. Liu, R. M. Osgood, Y. A. Vlasov, and M. J. GreenWilliam: Nature Photon 4, 557 (2010).

20. S. Zlatanovic, J. S. Park, S. Moro, J. M. C. Boggio, I. B. Divliansky, AlicNikola, S. Mookherjea, and S. Radic: Nature Photon 4, 561 (2010).

21. L. Shen, N. Healy, P. Mehta, T. D. Day, J. R. Sparks, J. V. Badding, and A. C. Peacock: Opt. Express 21, 13075 (2013).

22. F. Bai, Q. Wang, Z. Liu, X. Zhang, W. Lan, X. Tao, and Y. Sun: Appl. Phys. B 112, 83 (2013).

23. M. E. Fermann, V. I. Kruglov, B. C. Thomsen, J. M. Dudley, and J. D. Harvey: Phys. Rev. Lett 84, 6010 (2000).

24. M. J. McCarthy and D. C. Hanna: J. Opt. Soc. Am. B 10, 2180 (1993).

25. E. C. Cheung and J. M. Liu: J. Opt. Soc. Am. B 7, 1385 (1990).

26. O. Gayer, Z. Sacks, E. Galun, and A. Arie: Appl. Phys. B 91, 343 (2008).

27. R. Hegenbarth, A. Steinmann, G. Tóth, J. Hebling, and H. Giessen: J. Opt. Soc. Am. B 28, 1344 (2011).

28. M. Vainio, J. Peltola, S. Persijn, F. J. M. Harren, and L. Halonen: Appl. Phys. B 94, 411 (2009).

29. O. Kokabee, A. Esteban-Martin, and M. Ebrahim-Zadeh: Opt. Lett. 35, 3210 (2010).

30. D. S. Butterworth, S. Girard, and D. C. Hanna: Opt. Commun 123, 577 (1996).

31. A. C. Peacock, J. R. Sparks, and N. Healy: Laser \& Photonics Reviews 8, 53 (2014). 


\section{Figure and table captions}

Fig. 1. Schematic of the pump laser system and fs OPO: HWP, half-wave plate; L, lens; SMF, single mode fiber; CM, curved mirror; M, plane mirror; DM, dichroic mirror; HR, high-reflectivity mirror; LPF, long pass filter; PSD, position sensitive detector; BS, beam splitter; PZT, piezo translation stage.

Fig. 2. (a) Undepleted pump, depleted pump and depletion spectrum. (b) Signal and idler (2.4 $\mu \mathrm{m})$ output power vs. pump power.

Fig. 3. Variation of the average output power and wavelengths of signal and idler with OPO cavity length detuning.

Fig. 4. Variation of the idler pulse duration with wavelength. Inset is the intra-cavity group delay dispersion versus wavelength.

Fig. 5. Autocorrelation trace of the idler pulse at $2.4 \mu \mathrm{m}$. Inset: idler spectrum.

Fig. 6. M-squared measurement of idler in horizontal and vertical planes. Inset is the far-field idler beam profile.

Fig. 7. Idler output power observed over a 20 minute interval for unlocked and locked operation of the servo-loop stabilizing the OPO cavity length.

Fig. 8. Pump spectrum and supercontinuum generation in a 4-mm-long sample of silicon-core fiber for pump power of $15 \mathrm{~mW}$. 
Fig. 1.

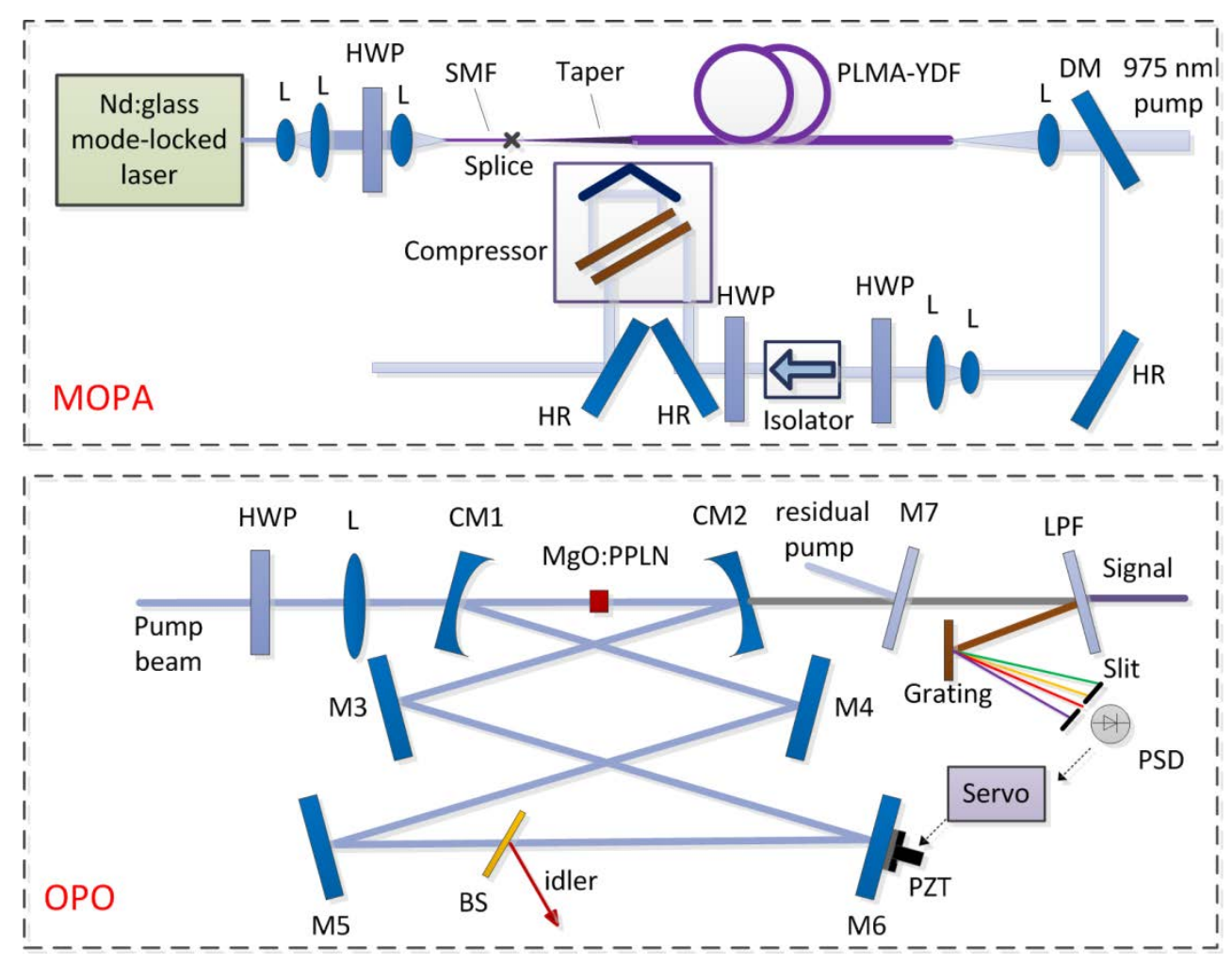


Fig. 2.
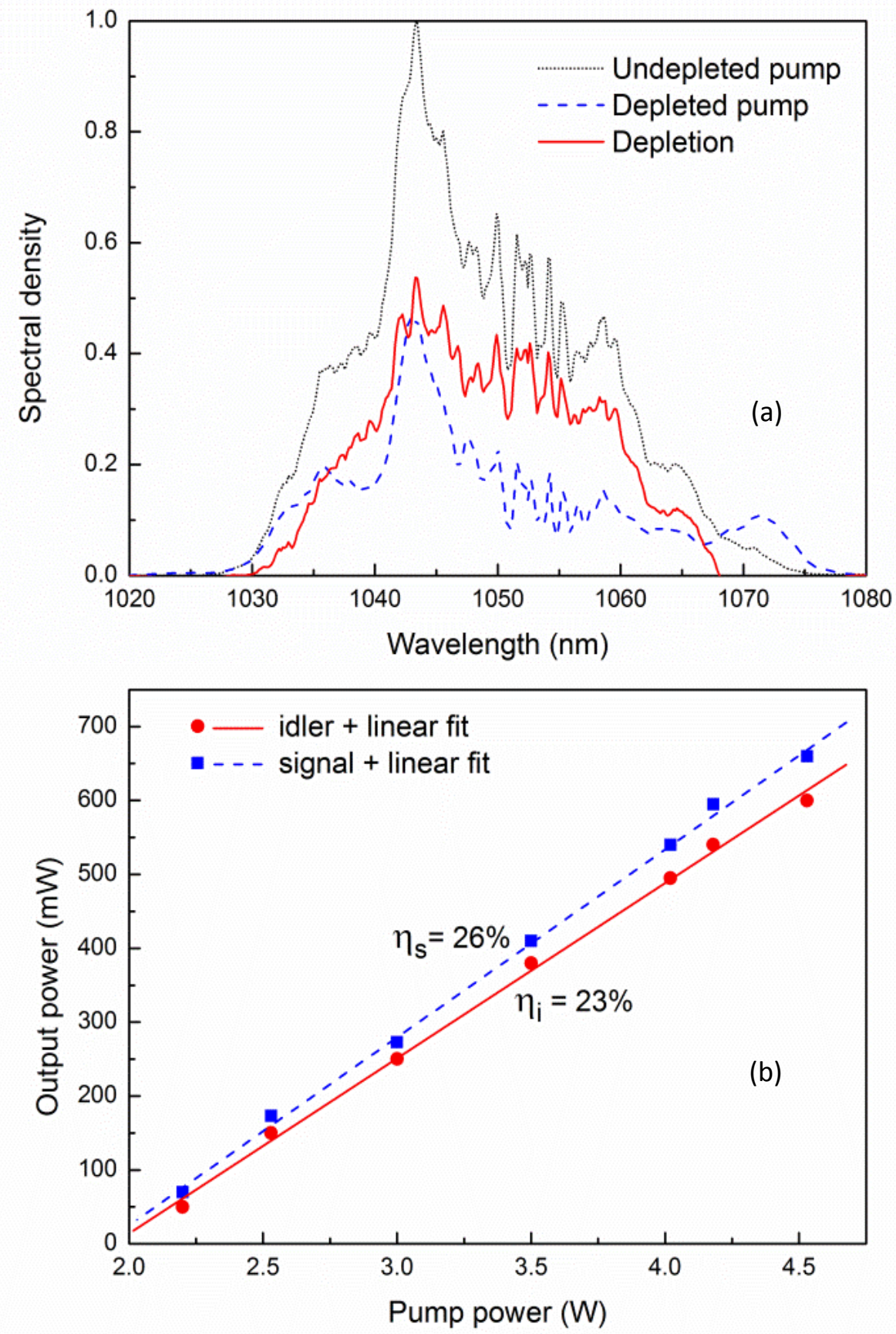
Fig. 3.

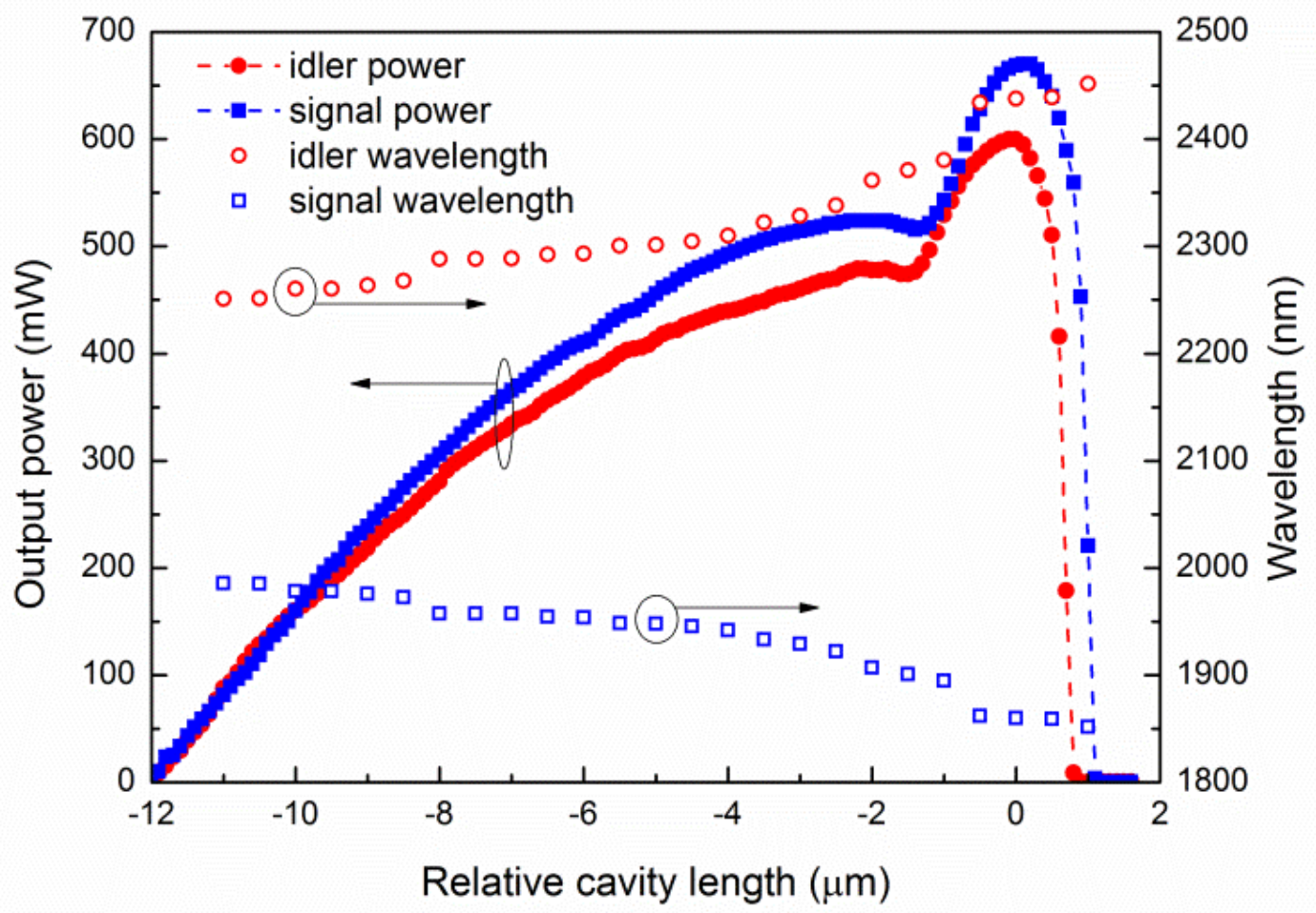


Fig. 4.

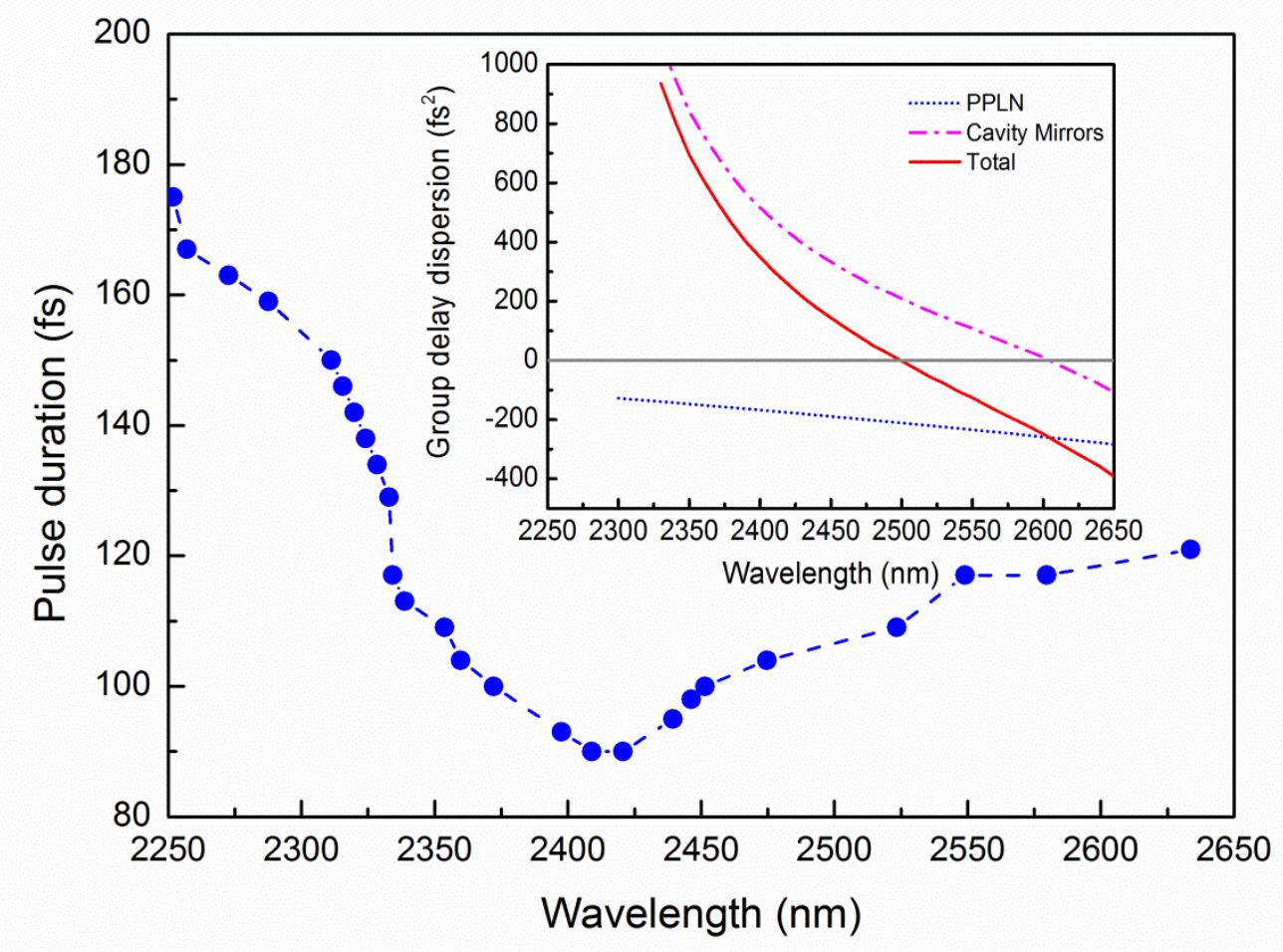


Fig. 5.

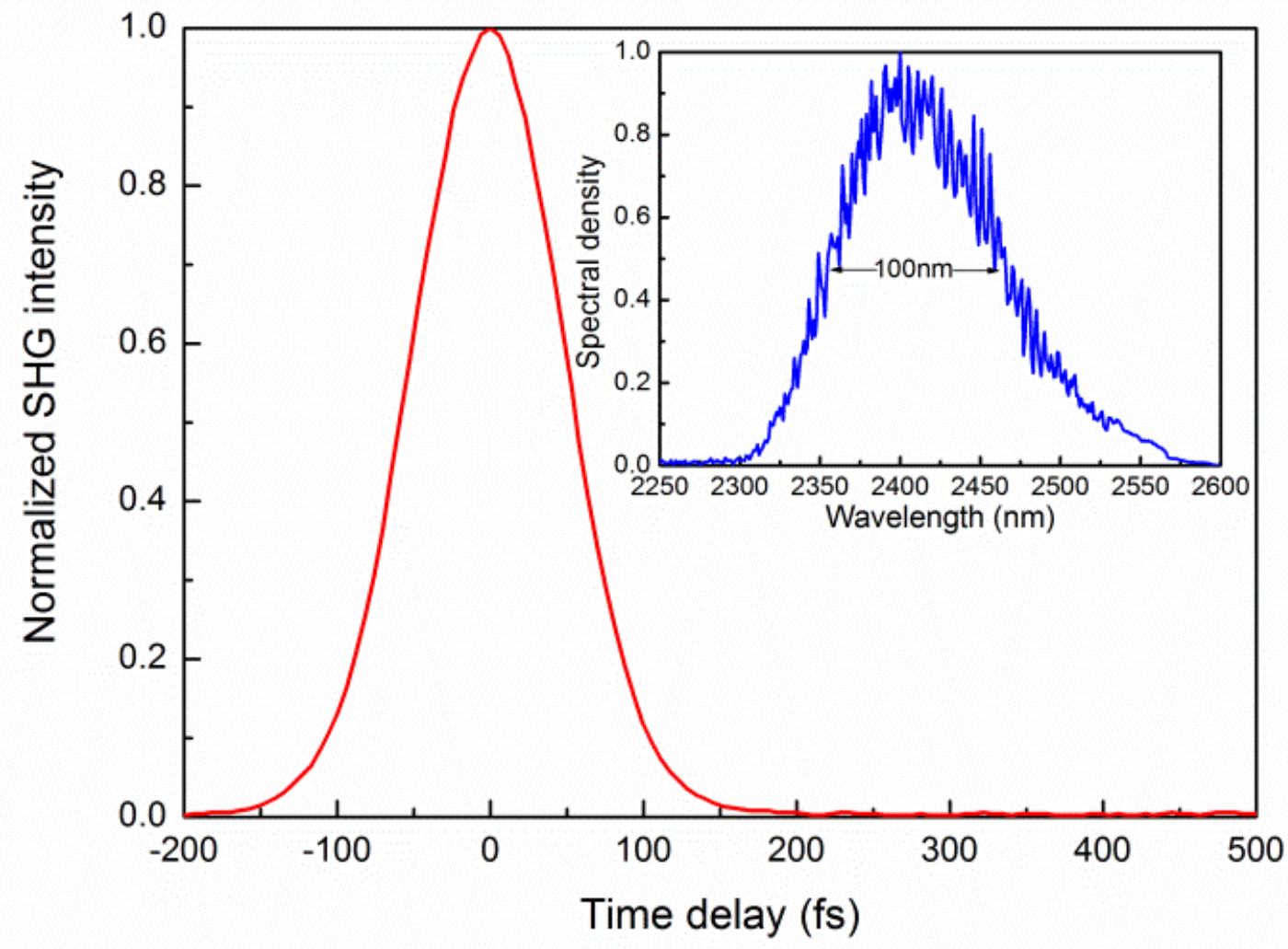


Fig. 6.

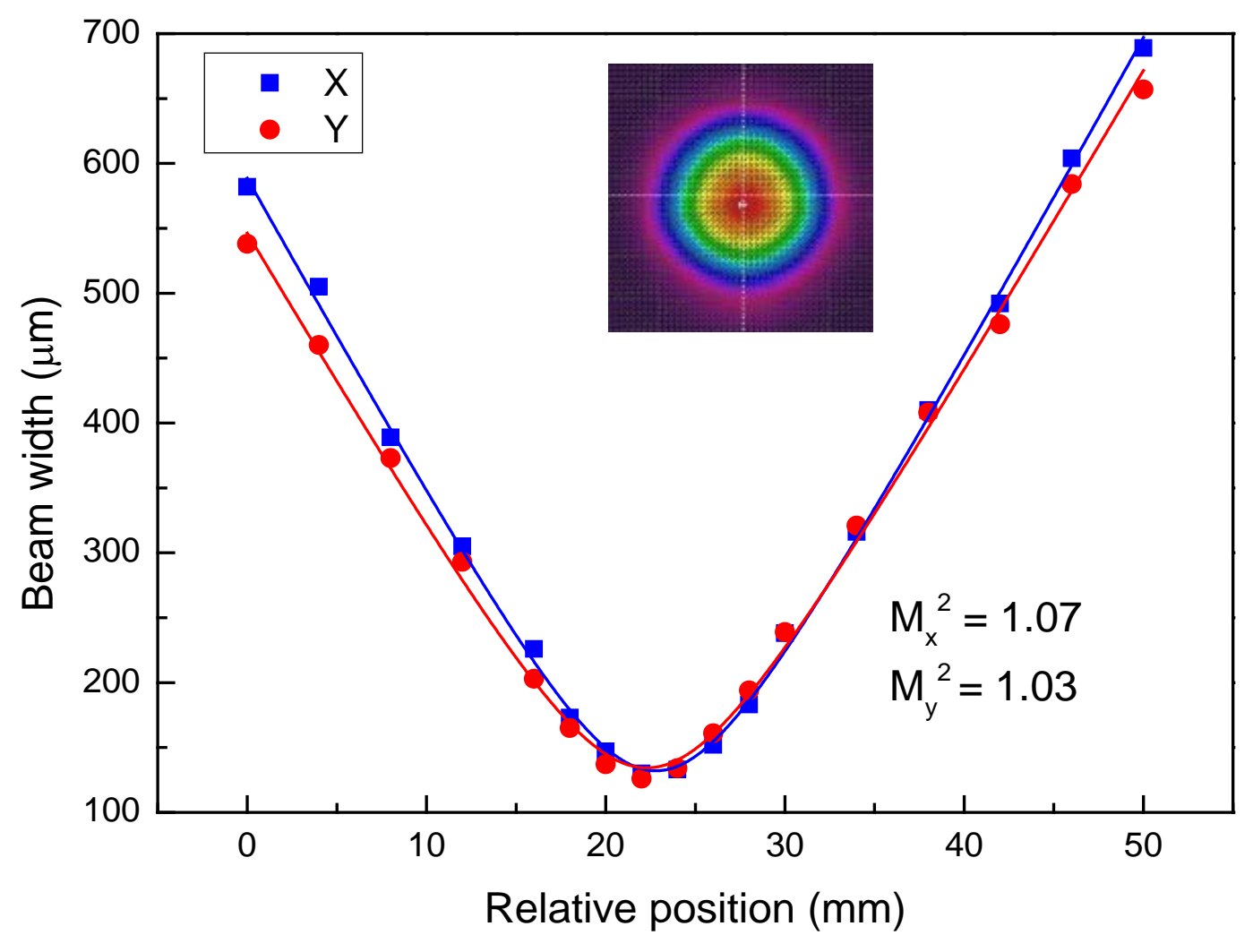


Fig. 7.

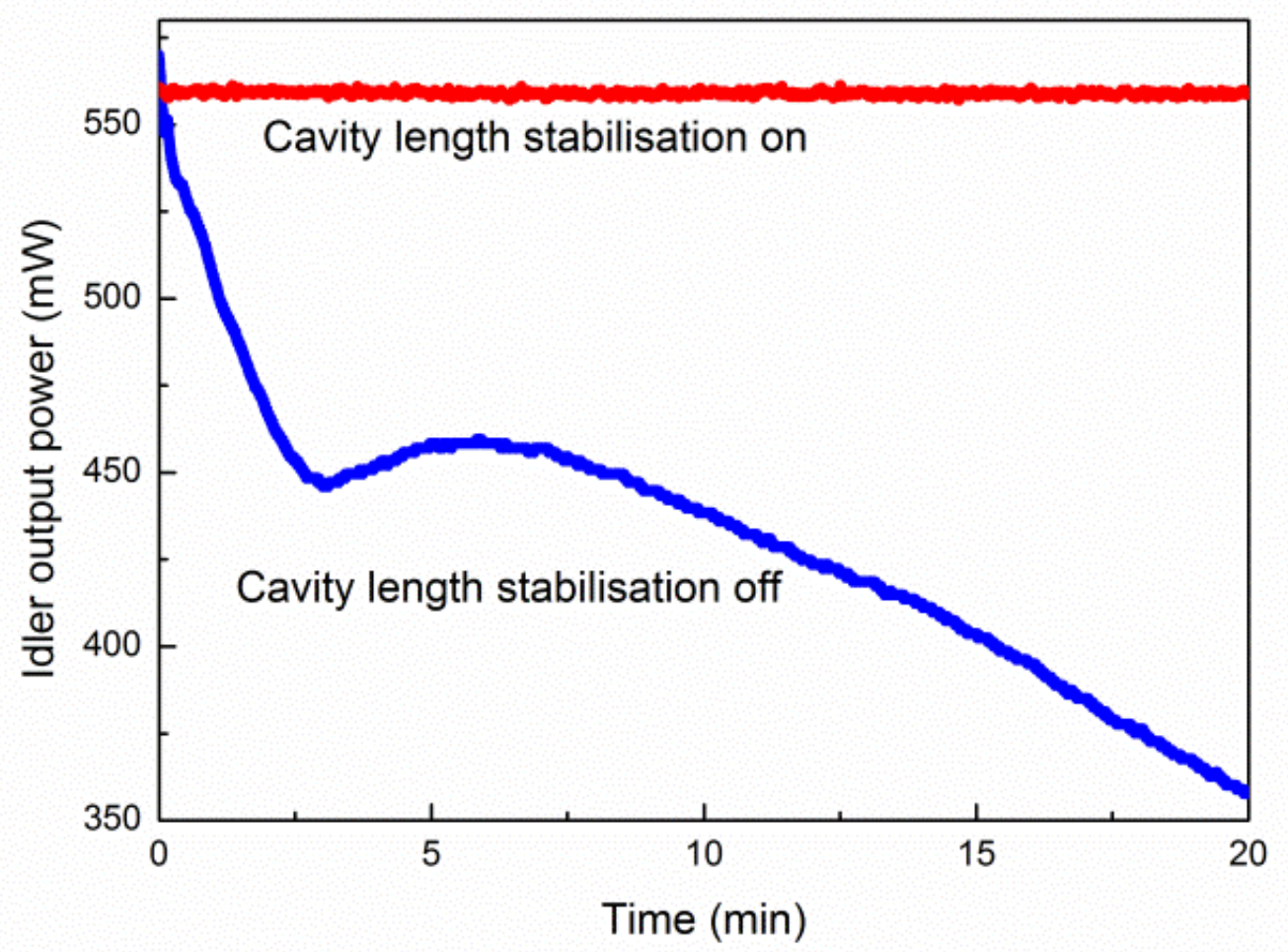


Fig. 8.

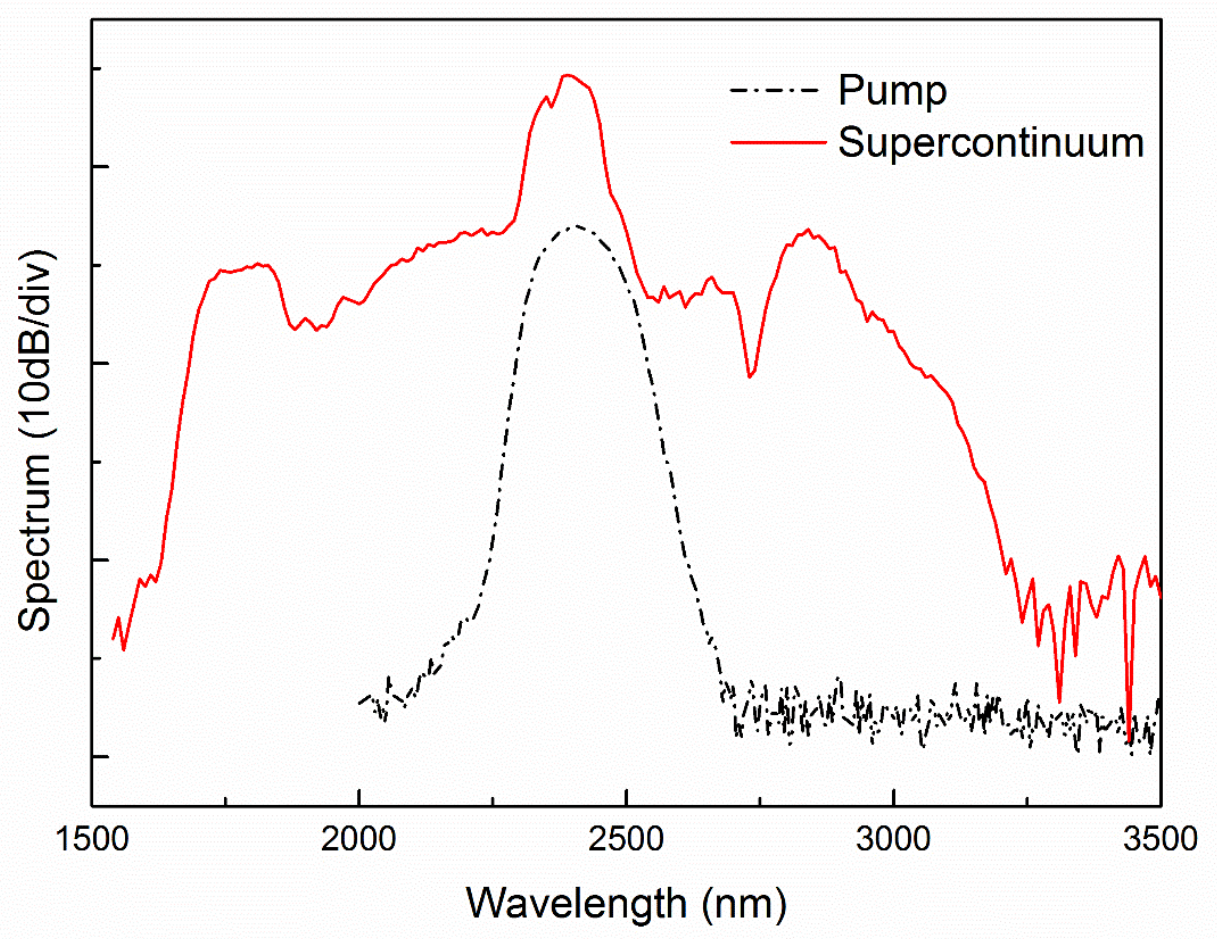

